\title{
Changing behavioral patterns to promote physical activity with motivational signs
}

\author{
Takuo Nomura · Yoshinobu Yoshimoto • \\ Yoshiteru Akezaki · Atushi Sato
}

Received: 3 March 2008/ Accepted: 11 September 2008/Published online: 25 October 2008

(c) The Japanese Society for Hygiene 2008

\begin{abstract}
Objectives We have evaluated the effectiveness of motivational signs in promoting stair use instead of escalators, in terms of gender and age, at a train station with a 37-step staircase. We also examined whether a newspaper article, or booster, had any effect on augmenting the impact of the signs.

Methods In a serial cross-sectional survey with prompted signs, the subjects were coded by gender and age (over 65 years, under 65 years), and students (13-18 years old and school uniforms). We used a 2-week baseline period, followed by a 4-week intervention in which a total of 45 posters and banners with motivational messages were used to encourage stair use. As a booster, we published a news release of this study on a web site and in a local newspaper during the intervention period.

Results A total of 43,241 escalator/stair-choice observations were made. Stair use increased significantly from 3.58 to $4.93 \%$ during the intervention period of $1-2$ weeks for all subjects. In addition, stair use had a significantly high value of $5.80 \%$ during the intervention period of 3-4 weeks. Stair use also increased in subgroups during the
\end{abstract}

T. Nomura $(\bowtie)$

Department of Physical Therapy,

School of Comprehensive Rehabilitation, Osaka Prefecture

University, 3-7-30 Habikino, Habikino, Osaka 583-8555, Japan

e-mail: nomurata@rehab.osakafu-u.ac.jp

Y. Yoshimoto · Y. Akezaki

Graduate School of Human Health Sciences,

Kochi Women's University, Kochi, Japan

A. Sato

Department of Health Science,

Faculty of Human Life and Environmental Science,

Kochi Women's University, Kochi, Japan intervention periods with the exception of women 65 and older. Attempts to publicize the study had no effect on the increase in stair use.

Conclusions The use of signs was effective in changing behavior during the period of sign use, but this effectiveness was limited by gender, age, and the physical ability (e.g., muscle strength) and energy required to climb the stairs. The possible synergistic effects of publicity were inconclusive but are worthy of further investigation.

Keywords Behavioral change - Exercise . Physical activity $\cdot$ Signs $\cdot$ Stair climbing

\section{Introduction}

Regular exercise has many positive benefits, including increases in maximum oxygen intake, decreases in myocardium oxygen consumption in certain submaximal loads, increased high-density lipoprotein cholesterol levels, and decreased triglyceride levels [1]. Type 2 diabetes is representative of a lifestyle-related disease where the onset and progression of the disease can be prevented by improved dietary habits and exercise regimes [2, 3]. It has also been consistently proven that appropriate exercise lowers the risk of chronic disease and leads to considerable public health gains. However, both the continuation of exercise and the maintenance of high levels of physical activity are highly difficult. Stair climbing is frequently available to most population groups and provides a useful model for lifestyle exercise interventions. From an operant conditioning perspective, the majority of people use an escalator when given a choice between an escalator and adjacent stairs [4]. The use of an escalator, rather than stairs, is reinforced because the escalator minimizes energy 
consumption and presents an easy alternative to muscle use. A previous study reported a change in unconscious decision-making to use the stairs instead of the escalator or elevator through intervention of motivational posters and/ or stair-riser banners. In other studies, the researchers specified the number of stairs [5-7, 9, 15, 16]. Motivational messages and paintings designed to boost stair use were printed on posters and stair-riser banners. In most of these studies, an increase in stair use was correlated with the use of these signs [5-15]. Kerr et al. [9] reported increased levels of stair use by a study cohort of elderly people in a shopping mall with an escalator and adjacent 24-step flight of stairs. In the surveys of Brownell et al. [5], Blamey et al. [6], Andersen et al. [7], and Webb et al. [15], the use of stairs of 8-30 steps, 15 steps, 10 steps, and 24 steps, respectively, were studied. In contrast, Eves et al. [16] reported that the effect of a signs was insignificant at an urban site with a travelator (i.e, an escalator without steps) and an adjacent 44-step flight of stairs.

We conducted a pilot study to promote stair use with stair-riser banners in the basement of a department store with an escalator and adjacent 14-step staircase [17]. Our results resembled those of the Kerr et al. study [9], and we concluded that signs were an effective means of promoting physical activity. However, the results of such studies should be interpreted with care: namely, we found that the effect of the signs was clearly evident on women-with the exception of elderly women and children [17]. In the Kerr et al. study [9], there was a significant correlation between the intervention and gender. In addition, it must be remembered that older people are physically less wellequipped to climb stairs because they have less leg strength than younger and middle-aged people. Therefore, Kerr et al. [9] evaluated the effectiveness of the signs in relation to gender, age, and number of steps to be climbed, but it is unclear whether similar effects could be achieved with a larger number of steps. In the study of Blamey et al. [6] and Kerr et al. [9], the overall stair use increased throughout the intervention period, then decreased gradually, even during the intervention period, as time passed. Titze et al. [13] reported that increased stair use could not be maintained after the removal of signs. After reviewing these studies, we believed it necessary to consider methods that would increase the strength of the message of the signs in promoting stair use. The Japanese Ministry of Health, Labour and Welfare recommends offering health information through various channels to increase healthy behavior among the general public. Healthy Japan 21 (a campaign to promote heath in the twenty-first century) [18] and an Exercise Guide 2006 [19] were disseminated through the mass media and Internet in Japan. However, the effect of utilizing the mass media and Internet in this manner remains to be thoroughly evaluated.
Our aim was to conduct a serial cross-sectional survey to measure the effectiveness of motivational posters and stair-riser banners to induce stair use versus escalator use. The study took place at a commuter train station in Japan and used a significantly larger number of stairs, in a longer staircase, than in previous studies. In addition, we assumed that press releases could be used to stimulate continued stair use. Hence, the secondary purpose of this study was to test the use of Internet and newspaper articles as measures to improve the effectiveness of this study.

\section{Methods}

\section{Setting}

The use of an escalator and the adjacent 37-step staircase use was monitored in a commuter train station in Himeji City, Hyogo Prefecture, Japan from 7:00 to 9:30 a.m. on two consecutive days per week, excluding weekends, from July through September, 2005 (Fig. 1). A total of 16 days were monitored during the study period. We used a total of 45 signs, which were placed on the stair risers (stair-riser Banners 1 and 2), a support pillar, and the side of the escalator (posters 1 and 2). Stair-riser banner 1 was $15 \times 120 \mathrm{~cm}$ (height $\times$ width; 17 banners) with an ultramarine-blue background; stair-riser banner 2 was $15 \times 75 \mathrm{~cm}$ (height $\times$ width; 17 banners) with a sky-blue background (Fig. 2). Poster 1 was $42 \times 59 \mathrm{~cm}$ (height $\times$ wide; six posters) with a green, yellow, or orange background, while poster 2 was $90 \times 90 \mathrm{~cm}$ (height $\times$ width; five posters) with a white background. Stair-riser banners 1 and 2 had 11 different messages: "Go up the stairs", "Exercise your legs", "Walk and be healthy", "Take the stairs", "For the prevention of disease", "Free exercise", "Exercise in daily life", "Exercise your heart", "Daily exercise", "Get more exercise", "Continuation is important", and "Well done!". The messages were printed in white. Poster 1 had six different messages: "After short periods of exercise, a high state of energy consumption is maintained.", "If you move your body frequently in daily life, you'll use more energy than you think.", "For example, use stairs, wash clothes. Do not use the TV remote control", "Repeated exercise produces positive metabolic results, such as healthy blood pressure, blood sugar levels and blood lipid levels". These messages were printed in black. The messages on poster 2 were simply "Walk," printed in large black letters in English.

This study was carried out with the permission and agreement of the train station. Video and facial photographs were not used. 
Fig. 1 Top-left photo Signs typically in place along the escalator and adjacent stairs, bottom-left photo the 45 signs (posters and banners with motivational messages) placed on alternate stair risers, pillars, and the wall, right schematic representation of the intervention site and the position of the observers

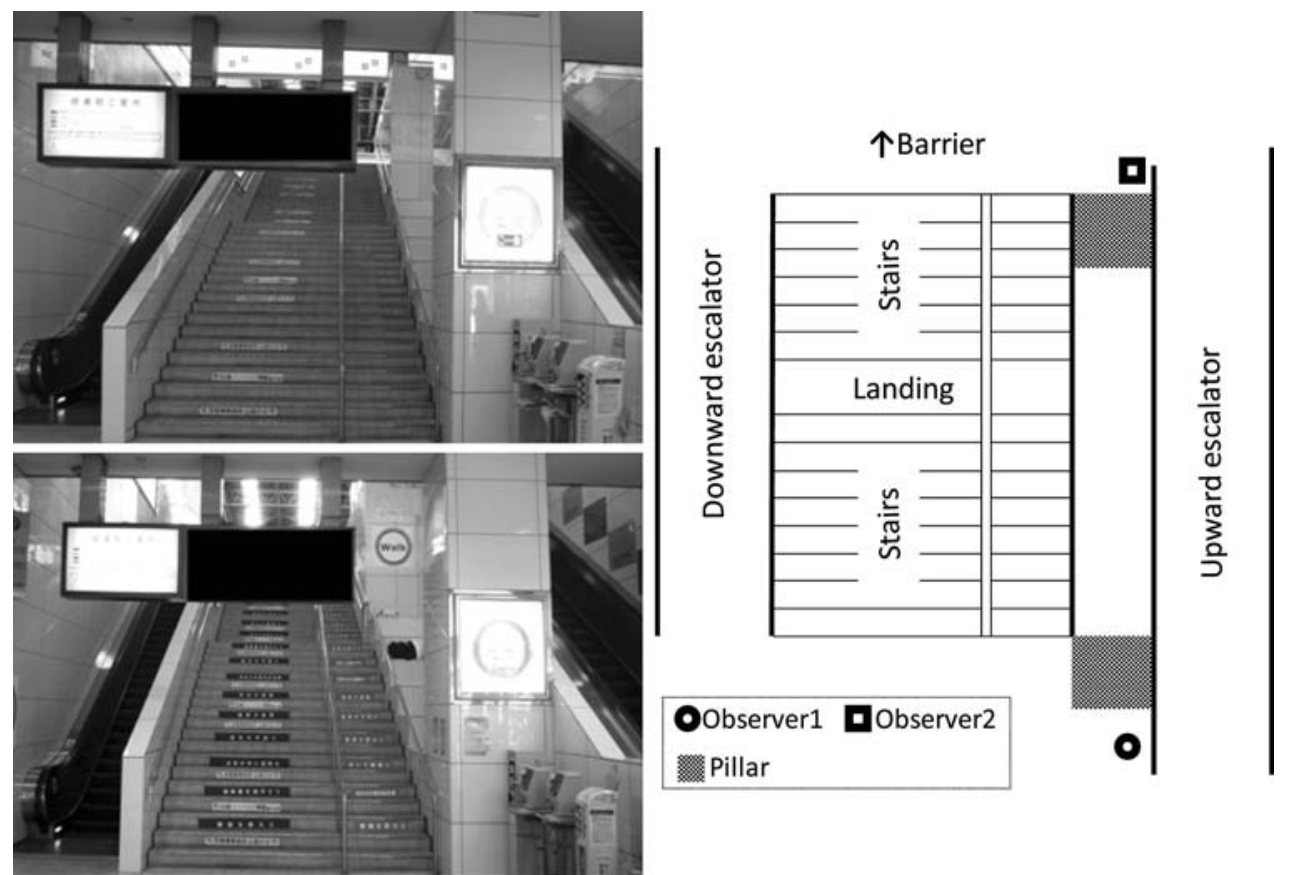

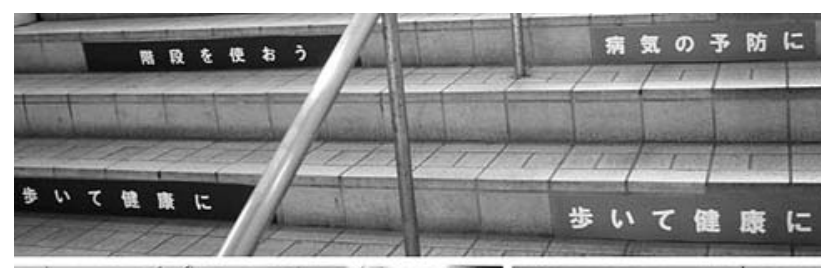
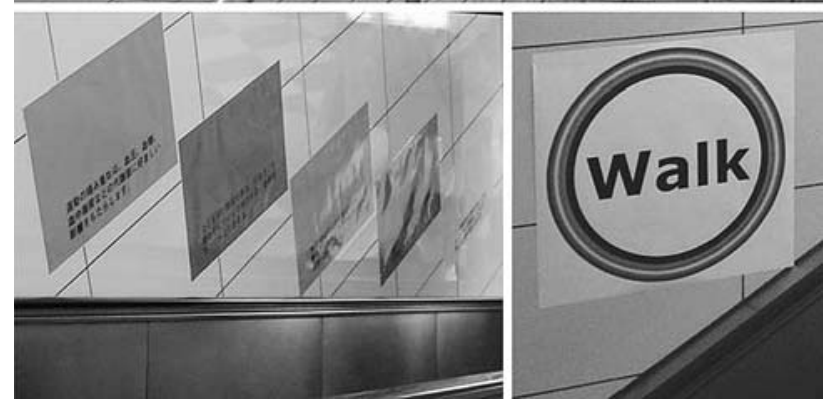

Fig. 2 An illustration of the signs: top photo stair-riser banners 1 and 2, bottom-left photo poster 1, bottom-right photo poster 2

\section{Procedures}

A serial cross-sectional survey was conducted by two inconspicuous observers. Both observers were seated in blind spots that were not visible to the subjects. Data were collected using a hand-counter. Observer 1 , positioned at the bottom of the stairs, recorded the number of people who climbed the stairs. Observer 2, positioned at the top of the stairs, counted the number of people who used the up escalator. The survey was conducted by a team from the same laboratory, and all observers had adequate training and practice prior to the study. The subjects were coded by gender and age (gray hair and/or an appearance of over
65 years old and gait) and as junior high school and high school students (wearing student uniforms and/or an appearance of 13-18 years old). Children (between waist and shoulder height of the accompanying adult) and infants were not included in the survey.

A 2-week baseline period was followed by a 4-week intervention using the signs. Follow-up data were collected during a 2-week period that took place 3 weeks after the removal of the signs. We used the Kobe Newspaper and its web site to publicize the study. An article was published on the web site on the fifth day of the third week of the intervention; a news release was published in the regional edition of the newspaper in the health column (size: $13.5 \times$ 22 , height $\times$ width) on the same day. The Kobe Newspaper is the major local newspaper in Hyogo Prefecture; the morning edition had a local circulation of 568,687 , or approximately $30 \%$ of the local population, in 2005 .

\section{Statistical analysis}

The intervention and follow-up periods were compared to the baseline period using chi-square analysis and logistic regression analysis: a logistic regression analysis was applied to escalator or stair use as a dichotomous outcome variable (coded as escalator use $=1$, stair use $=2$ ). Signs (coded as no signs $=0$, signs use $=1$ ) were incorporated as independent variables for a logistic regression analysis of the outcome variables. In addition, the pre- and postbooster data for the newspaper article and the 3-day period preceding and following the article were compared using chi-square analysis. 
Statistical analysis was conducted using the SPSS statistical program, ver. 15.0 J (SPSS, Chicago, IL), and statistical significance was defined as $P<0.05$.

\section{Results}

A total of 43,241 escalator- or stair-choice observations were made during the study periods. The subjects were categorized by gender and age. There were 1670 women in the 65-year-and-older group, 16,687 women in the under65-year group, and 2797 female students. There 1728 men in the 65-year-and-older group, 17,631 men in the under65-year group, and 2728 male students. The pedestrian traffic volume had a mean value of $5405.1 \pm 292.1$ per week, or a coefficient of variation of $5.4 \%$.

Stair use increased significantly from 3.58 to $4.93 \%$ [odds ratio (OR) 1.39, 95\% confidence interval (CI) 1.221.59] during the intervention periods of 1-2 weeks for all subjects. In addition, stair use had a significantly high value of $5.80 \%$ (OR $1.56,95 \%$ CI 1.36-1.77) during the intervention periods of 3-4 weeks. When the signs were removed, stair use returned to the baseline value. Figure 3 shows the percentage of stair use and the specific and overall distributions for the periods before, during, and after the intervention periods by gender and age. Table 1 shows the OR and 95\% CI for stair use compared to baseline by gender and age. During the intervention period of 1-2 weeks, the percentage of people who used the stairs increased significantly, from 0.39 to $4.15 \%$ (OR $11.0,95 \%$ CI 1.47-81.92) for men 65 years and older, from 3.93 to $20.60 \%$ (OR 6.33, 95\% CI 4.12-9.71) for female students, and from 10.75 to $17.08 \%$ (OR $1.70,95 \%$ CI $1.22-2.37$ ) for male students. During the intervention period of 34 weeks, the use of stairs by the under-65-year group also increased significantly, from 2.57 to $3.46 \%$ (OR 1.35 , CI 1.04-1.76) for women and from 3.86 to $5.07 \%$ (OR 1.32 , CI 0.08-1.63) for men. In addition, the percentages for stair use during the intervention period of 3-4 weeks were $2.85 \%$ (OR 7.46, 95\% CI 0.99-55.74) for men 65 years and older, $10.11 \%$ (OR 2.74, 95\% CI 1.76-4.26) for female students, and $17.89 \%$ (OR 1.80, 95\% CI 1.32-2.47) for male students. These values are significantly above baseline levels. During the follow-up periods, the percentages for stair use, in comparison with the baseline, were significantly higher only for the 65-year-and-older group.

The newspaper and Internet articles clearly did not have any effect on the findings: namely, stair use before and after the press releases was 6.46 and $4.89 \%$, respectively. Stair use for the 3-day period preceding and following the press releases was 5.69 and $4.10 \%$, respectively. Similarly, the press releases had no effect when applied to subjects by gender or age.
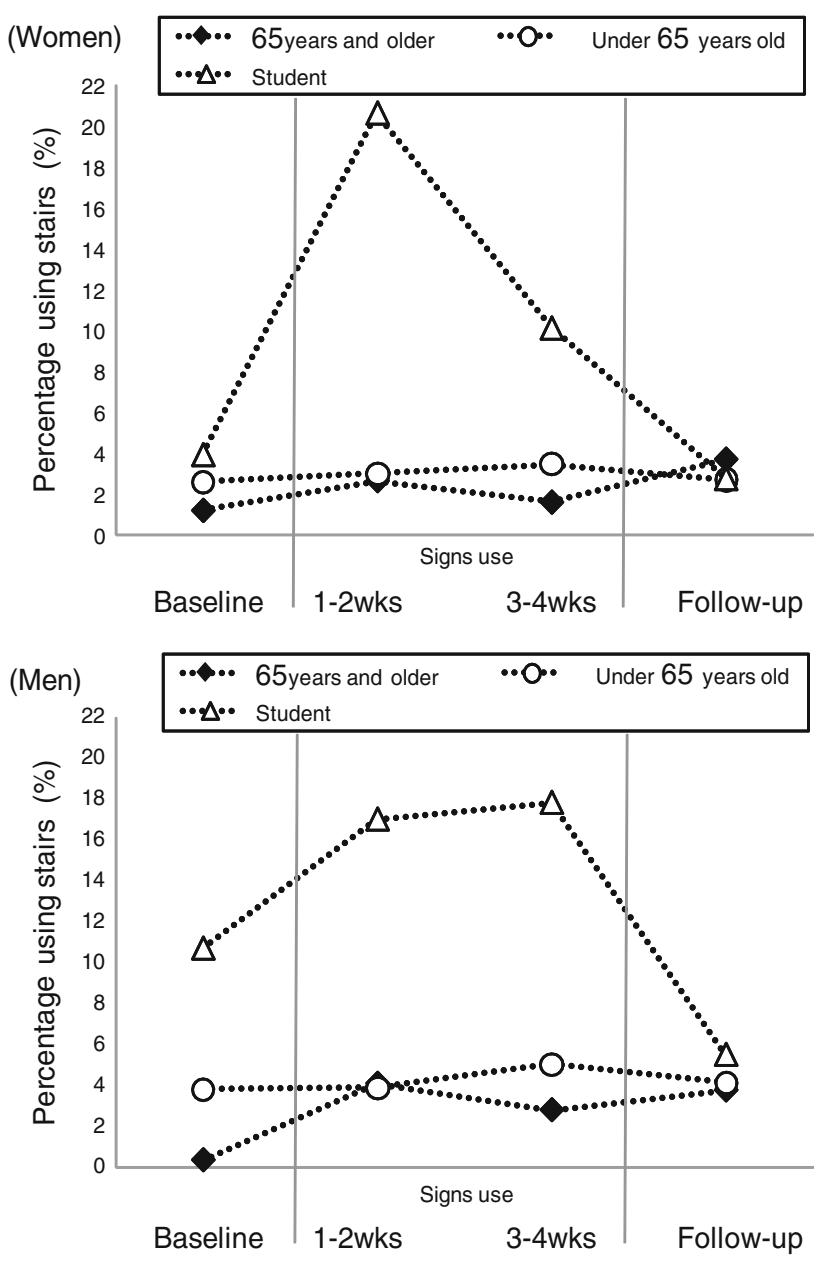

Fig. 3 Percentage of stair use during and after sign use by gender and age. Top Data on women, bottom data on men

\section{Discussion}

We have examined the effectiveness of signs in encouraging stair use over escalators at a train station with a 37-step staircase. The gross caloric "cost" of stair use (i.e., ascending and descending a step) is $0.16-0.237 \mathrm{kcal} \mathrm{step}^{-1}$ [20]. Calorie consumption is magnified by the number of stairs that are climbed or descended. Long staircases with multiple motivational signs would seem to be more effective in burning calories. In this study, stair use increased significantly for men 65 and older and students during the 1-2 week period of sign use. Students in particular reacted strongly to the signs, and their stair use increased by more than $150 \%$. Students may have been the group for which the behavior change was the easiest, since they are, in general, physically capable of climbing stairs; however, we were unable to determine a cause for this behavioral change. Conversely, stair use significantly decreased for male students after the motivational banners were removed. The factors affecting these behavioral changes will be examined in future studies. Stair use also increased 
Table 1 Stair use as a result of motivational signs compared to baseline by gender and age

\begin{tabular}{|c|c|c|c|c|c|c|}
\hline \multirow[t]{2}{*}{ Study cohort } & \multicolumn{3}{|c|}{ Women } & \multicolumn{3}{|l|}{ Men } \\
\hline & OR & $95 \% \mathrm{CI}$ & $P$ value & OR & $95 \% \mathrm{CI}$ & $P$ value \\
\hline \multicolumn{7}{|l|}{65 years and older } \\
\hline Baseline versus sign use $1-2$ weeks & 2.23 & $0.80-6.18$ & 0.12 & 11.00 & $1.47-81.92$ & $<0.01$ \\
\hline Baseline versus sign use $3-4$ weeks & 1.33 & $0.44-4.02$ & 0.60 & 7.46 & $0.99-55.74$ & $<0.05$ \\
\hline Baseline versus follow-up & 3.13 & $0.89-11.01$ & 0.07 & 10.10 & $1.23-82.83$ & $<0.05$ \\
\hline \multicolumn{7}{|l|}{ Under 65 years old } \\
\hline Baseline versus sign use $1-2$ weeks & 1.16 & $0.90-1.51$ & 0.24 & 1.02 & $0.83-1.26$ & 0.82 \\
\hline Baseline versus sign use $3-4$ weeks & 1.35 & $1.04-1.76$ & $<0.05$ & 1.32 & $0.08-1.63$ & $<0.01$ \\
\hline Baseline versus follow-up & 1.04 & $0.80-1.36$ & 0.74 & 1.08 & $0.88-1.33$ & 0.43 \\
\hline \multicolumn{7}{|l|}{ Student } \\
\hline Baseline versus sign use $1-2$ weeks & 6.33 & $4.12-9.71$ & $<0.001$ & 1.70 & $1.22-2.37$ & $<0.001$ \\
\hline Baseline versus sign use $3-4$ weeks & 2.74 & $1.76-4.26$ & $<0.001$ & 1.80 & $1.32-2.47$ & $<0.001$ \\
\hline Baseline versus follow-up & 0.69 & $0.40-1.19$ & 0.19 & 0.48 & $0.32-0.73$ & $<0.001$ \\
\hline
\end{tabular}

OR Odds ratio; $\mathrm{CI}$, confidence interval

significantly in the under-65-year group as a whole during the 3-4 week period of sign use, although the use of the signs did not correspond to a significant increase in stair use by women 65 years and older. This is in contrast to the results of an earlier study that used a 14-step flight of stairs [17] in which we were able to demonstrate the effectiveness of a signs for increasing stair use in women 65 years and older. In addition, Kerr et al. [9] reported the effectiveness of a signs in increasing stair use among aged women when using a 24-steps flight of stairs. When leg strength falls below a specific standard, physical functional performance becomes difficult [21]. Declining leg strength, due to aging, impacts on choices to use stairs. Muscle strength could be a factor in choosing to climb a long flight of stairs rather than using an escalator or elevator. Women generally have less leg strength than men of the same age group. Hence, women use a larger ratio of muscle strength when climbing stairs. This study demonstrates that the efficiency of promoting stair use with signs is limited by gender and age based on an individual's physical condition: namely, older individuals (particularly older woman) will more readily climb shorter flights of stairs and tend to avoid climbing longer flights of stairs.

Sedentary behavior causes and prolongs obesity [22] and is a risk factor in many diseases. In addition, despite decreases in death rates from chronic diseases, such as obstructive pulmonary disease and diabetes mellitus [23], the total number of deaths is increasing, although these deaths occur at older ages. Manini et al. reported that energy expenditure due to normal daily activity was strongly associated with a lower risk of mortality in older people [24]. Simply expending energy through any activity, therefore, may influence survival rates. It has been proven that signs are an effective method of promoting stair use among older people for staircases of approximately 20 steps $[12,17]$. However, based on the results of this study, we must consider the limits placed by exercise loads and strength levels when we target an older population for behavioral change. Moreover, we did not examine the effects of using a different message content, colors, or size for the signs or the effect of differences in location. These areas remain a subject for a future study.

Our secondary aim was to measure the effects of positive publicity in local media to boost stair use. However, we were unable to find any effects. We initially thought that articles on web pages or in local newspapers would raise public awareness of both the studies and exercise options in daily life, but we found that the effect of the press releases used in this study was negligible in promoting stair use. The main determinant may have been that most subjects simply did not read the press releases. Another factor may have been that the column size of the article was too small and not noticed by many people. In addition, the contents of the article may have failed to generate reader interest or motivation to climb the stairs. A future study will consider the use of various types of media and deployment strategies in order to examine how they influence behavior when used in conjunction with behavioral change projects.

In our previous study, stair use did not increase immediately after the motivational signs were introduced [17]. The use of stairs increased during the intervention periods of 1-2 weeks in men 65 years and older and in both student groups. Stair use by female students, on the other hand, tended to drop during the intervention periods of the third and fourth weeks when compared with the data for weeks one and two. We were unable to conclude whether the effectiveness of the signs was due to the initial recognition 
or repeat viewing. Further investigation of this point is therefore necessary. We also did not examine the reliability of the observational methods and their effect on accuracy. In addition, our study design did not include a control and also shows only a short-term effect. We were able to change behavior in an intervention location, but it was unclear whether the behavior is generalized and would have similar effects in other locations. These are the limits to our study. Many types of personal behavior may be modified by using signs placed at various locations. If physical activity is repeatedly prompted and encouraged, calorie use is increased, and lifestyle-related diseases can be prevented. Motivational signs are effective, even in the case of large numbers of stairs, but behavioral change is limited by gender and age.

Acknowledgments This study was partially supported by a grant to encourage young researchers from The Japanese Society for Hygiene. The authors thank Prof. Hiroyuki Nakamura of the Department of Environmental and Preventive Medicine, Graduate School of Medical Science, Kanazawa University, who provided valuable advice and completed the analyses. The authors also thank Craig Delaney, Yutaka Tomita, Ryo Fujiwara, Takashi Hamakubo, and Takeo Higashi for assisting with this study, and the management of site for assistance in conducting this survey.

\section{References}

1. Hahn RA, Teutsch SM, Rothenberg RB, Marks JS. Excess deaths from nine chronic diseases in the United States, 1986. JAMA. 1990;264:2654-9.

2. Eriksson KF, Lindgarde F. No excess 12-year mortality in men with impaired glucose tolerance who participated in the Malmo preventive trial with diet and exercise. Diabetologia. 1998;41: 1010-6.

3. Lindström J, Louheranta A, Mannelin M, Rastas M, Salminen V, Eriksson J, et al. and the Finnish Diabetes Prevention Study Group (2003) The Finnish diabetes prevention study (DPS): lifestyle intervention and 3-year results on diet and physical activity. Diabetes Care 26:3230-6.

4. Aarts H, Paulussen T, Schaalma H. Physical exercise habit: on the conceptualization and formation of habitual health behaviours. Health Educ Res. 1997;12(3):363-74.

5. Brownell KD, Stunkard AJ, Albaum JM. Evaluation and modification of exercise patterns in the natural environment. Am J Psychiatry. 1980;137:1540-5.

6. Blamey A, Mutrie N, Aitchison T. Health promotion by encouraged use of stairs. Br Med J. 1995;29:289-90.

7. Andersen RE, Franckowiak SC, Snyder J, Bartlett SJ, Fontaine KR. Can inexpensive signs encourage the use of stairs? Results from a community intervention. Ann Intern Med. 1998;129:363-9.
8. Russell WD, Dzewaltowski DA, Ryan GJ. The effectiveness of a point-of-decision prompt in deterring sedentary behavior. Am J Health Promot. 1999;13:257-9.

9. Kerr J, Eves F, Carroll D. Six-month observational study of prompted stair climbing. Prev Med. 2001;33:422-7.

10. Coleman KJ, Gonzalez EC. Promoting stair use in a US-Mexico border community. Am J Public Health. 2001;91:2007-9.

11. Boutelle KN, Jeffery RW, Murray DM, Schmitz MK. Using signs, artwork, and music to promote stair use in a public building. Am J Public Health. 2001;91:2004-6.

12. Kerr J, Eves F, Carroll D. Encouraging stair use: stair-riser banners are better than posters. Am J Public Health. 2001;91:1192-3.

13. Titze S, Martin BW, Seiler R, Marti B. A worksite intervention module encouraging the use of stairs: results and evaluation issues. Soz Praventiv Med. 2001;46:13-9.

14. Marshall AL, Bauman AE, Patch C, Wilson J, Chen J. Can motivational signs prompt increases in incidental physical activity in an Australian health-care facility? Health Educ Res. 2002;17:743-9.

15. Webb OJ, Eves FF. Promoting stair use: single versus multiple stair-riser messages. Am J Public Health. 2005;95:1543-4.

16. Eves FF, Masters RS. An uphill struggle: effects of a point-ofchoice stair climbing intervention in a non-English speaking population. Int J Epidemiol. 2006;35:1286-90.

17. Nomura T, Enoki H, Okazaki R, Sato A. Promoting daily physical activity by encouraging stair using banners (in Japanese). Nippon Eiseigaku Zasshi. 2006;61:38-43.

18. The Office for Lifestyle-Related Diseases Control (2000) General Affairs Division, Health Service Bureau, Ministry of Health, Labour and Welfare of Japan. Japanese National Health Promotion in the 21st Century (Healthy Japan 21). Available at: http://dailywify. googlepages.com/p00e-HealthyJapan21 generalstatement.pdf. Accessed 11 July 2008.

19. The Office for Lifestyle-Related Diseases Control (2006) General Affairs Division, Health Service Bureau, Ministry of Health, Labour and Welfare of Japan. Exercise and Physical Activity Guide for Health Promotion 2006 (Exercise Guide 2006). Available at: http://www.nih.go.jp/eiken/programs/pdf/exercise_ guide.pdf. Accessed 11 July 2008.

20. The KC, Aziz AR. Heart rate, oxygen uptake, and energy cost of ascending and descending the stairs. Med Sci Sports Exerc. 2002;34:695-9.

21. Cress ME, Meyer M. Maximal voluntary and functional performance levels needed for independence in adults aged 65 to 97 years. Phys Ther. 2003;83:37-48.

22. Ravussin Eric. A neat way to control weight? Science. 2005;307:530-1.

23. Jemal A, Ward E, Hao Y, Thun M. Trends in the leading causes of death in the United States, 1970-2002. JAMA. 2005; 294:1255-9.

24. Manini TM, Everhart JE, Patel KV, Schoeller DA, Colbert LH, Visser M, et al. Daily activity energy expenditure and mortality among older adults. JAMA. 2006;296:216-8. 